\title{
Phronesis
}

\section{Professionnaliser les acteurs de l'éducation et de la formation : entre construction identitaire et logiques de déprofessionnalisation}

\section{Philippe Maubant et Stéphane Martineau}

Volume 1, numéro 2, avril 2012

URI : https://id.erudit.org/iderudit/1009062ar

DOI : https://doi.org/10.7202/1009062ar

Aller au sommaire du numéro

Éditeur(s)

Institut de recherche sur les pratiques éducatives

ISSN

1925-4873 (numérique)

Découvrir la revue

Citer cette note

Maubant, P. \& Martineau, S. (2012). Professionnaliser les acteurs de l'éducation et de la formation : entre construction identitaire et logiques de

déprofessionnalisation. Phronesis, 1(2), 89-92.

https://doi.org/10.7202/1009062ar d'utilisation que vous pouvez consulter en ligne. 


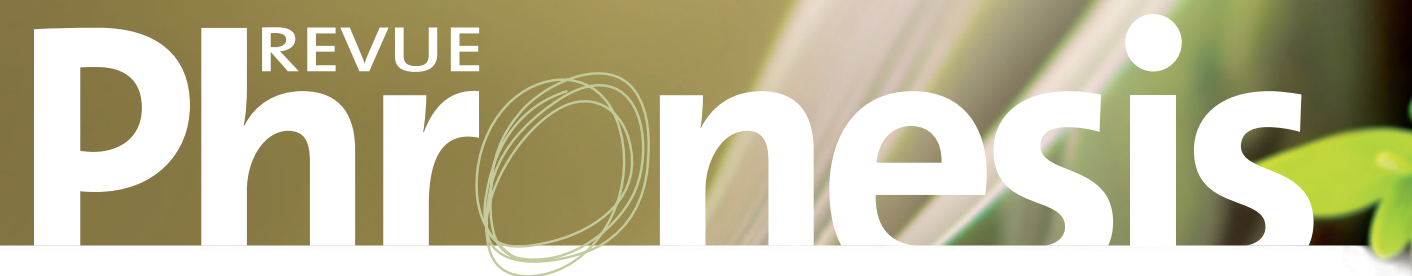

Institut de recherche sur les pratiques éducatives de l'Université de Sherbrooke

\section{Professionnaliser les acteurs de léducation et de la formation : entre construction identitaire et logiques de déprofessionnalisation \\ Philippe Maubant* et Stéphane Martineau**}

*Institut de recherche sur les pratiques éducatives

Université de Sherbrooke

2500 boul de l'Université Sherbrooke, Québec, Canada, J1K 2R1

Philippe.Maubant@USherbrooke.ca

${ }^{*}$ Laboratoire du développement et de 'INsertion professionnelle en enseignement

Université du Québec à Trois-Rivières

C.P. 500 Trois-Rivières, Québec, Canada, G9A 5H7

stephane.martineau@uqtr.ca

Note de synthèse 
Mais les situations et processus de construction identitaire se doivent dềtre étudiés au regard des types de dispositifs de formation professionnelle dans lesquels ils se déploient. Sur les traces proposées par Pelanda Dieci, Weiss \& Monnier, nous pouvons admettre que les processus de construction identitaire se nourrissent des différentes situations potentiellement formatives rencontrées par les professionnels en devenir. Ici, la problématique de l'alternance en formation rejoint celle de l'identité professionnelle. Ainsi, nous pouvons considérer que les caractéristiques des différentes situations potentiellement formatives rencontrées par les enseignants en formation, notamment pendant les périodes de stages, influencent les conditions et modalités de construction et de développement de l'identité professionnelle. Nous considérons en effet que les concepteurs de dispositifs de formations professionnelles font le pari de proposer différentes situations formatives selon des temporalités et des contextes variés, mais qu'ils ne vérifient pas nécessairement que ces situations créent les conditions d'un apprentissage professionnel.

Décrire et analyser les dispositifs, situations et processus de professionnalisation, c’est aussi lire, comprendre et interpréter les discours « sur et pour " la professionnalisation. Certains textes parus autour de cette problématique de la professionnalisation sont marqués par l'abondance, voire l'abus de concepts et de théories, souvent peu explicités et dont les fondements et références sont parfois incertains. Dans ce contexte conceptuel opaque et ce florilège d'allants-de-soi, la réflexivité et/ou l'analyse réflexive tient le haut du pavé. Les démarches et les outils pour créer ou favoriser de la réflexivité sont nombreux. L’observation d'un professionnel en situation, les activités de rétroaction, l'enregistrement par vidéo d'un professionnel en activité sont autant de dispositifs visant à créer les conditions de la réflexivité. Or, il semble important, à l'instar de Derobertmasure \& Dehon, de s'interroger sur le sens de ces dispositifs et sur les intentions des zélateurs de l'analyse réflexive. Ces deux auteurs nous invitent d'ailleurs pertinemment à questionner les fondements de la réflexivité au regard de deux finalités : la recherche et la formation. Nous pouvons constater ici combien les recherches sur les pratiques enseignantes, voire sur les pratiques d'enseignement, visent prioritairement aujourd'hui la mise en évidence de bonnes pratiques. Il s'agit de montrer l'efficacité de tel ou tel dispositif didactico-pédagogique. Dès lors, prendre pour objet de recherche la pratique d'enseignement, c'est se situer sur une délicate ligne de partage et de tension entre deux finalités : une visée de production de savoirs scientifiques et une visée de formation. L’objectif de former des praticiens réflexifs est aujourd'hui omniprésent dans bon nombre de curriculum de formation à l'enseignement. Mais convoquer le regard du chercheur pour analyser le développement de processus réflexifs en formation, c'est accepter de remettre en question le dogme de la réflexivité. C'est décrire et comprendre les différentes situations formatives pouvant créer les conditions d'une «véritable» réflexivité (Buysse, 2011). C'est enfin prendre le risque d'interroger la finalité intrinsèque de la réflexivité dans la conception et dans la mise en ouvre de l'activité professionnelle.

Les différents textes présentés dans ce numéro 2 de la revue Phronesis nous invitent donc à débattre des questions de construction et de développement identitaire dans les métiers adressés à autrui. Ils nous guident sans doute vers une dimension d'analyse de la professionnalisation, celle qui vise à relier la question du sens de la professionnalisation à celle des caractéristiques des différentes situations qui contribuent ou non à cette professionnalisation. Par exemple, si Roquet nous rappelle la nécessité d'interroger la professionnalisation à partir de trois niveaux d’analyse, il nous encourage aussi à envisager les possibilités d'une déprofessionnalisation qui pourrait se révéler principalement par l’observation des situations et processus d'élaboration, voire de modifications de l'identité professionnelle. Les processus de construction identitaire ne constituent pas un long fleuve tranquille conduisant le professionnel en devenir à un professionnel en maitrise de compétences et affichant avec arrogance et détermination sa professionnalité. Lobservation et l’analyse de ces processus identitaires peuvent aussi révéler la fragilité des situations professionnelles, la violence des contextes de travail, les occasions de non- reconnaissance, la précarité des emplois, la déqualification. Ces signes traduisent des crises, voire des fractures identitaires. Ils peuvent révéler des atteintes irréversibles à l'identité même du sujet, le conduisant progressivement d'une déqualification vers une déprofessionnalisation.

Les pressions imposées à l’éducation, comme à la santé ou à l'action sociale, par les politiques budgétaires des États expliquent en grande partie aujourd'hui cette obligation de résultats. L’activité des métiers adressés à autrui se trouve interrogée dans sa capacité à répondre aux grandes problématiques sociales. Mais elle est aussi évaluée au regard de ce qu'elle coute aux contribuables et de ce qu'elle rapporte aux États. Derrière certains discours sur et pour la professionnalisation, deux doctrines semblent coexister: la première est celle du soupçon à l'égard des professionnels de l'éducation et de la formation, suspectés de " mal " travailler. La seconde est celle du credo néolibéral rappelant avec insistance le coût des dépenses publiques d'éducation.

En somme, on peut lire les contributions de ce numéro comme autant d'invitations à la prudence face à l’omniprésence des discours sur la profes- 
sionnalisation. Ils cherchent en quelque sorte à nous alerter au sujet de l'accroissement de l'écart entre des propos enchanteurs sur la professionnalisation et des réalités professionnelles complexes où le triomphalisme nést de toute évidence pas de mise. Ils nous invitent enfin à faire preuve d'une grande vigilance dans nos analyses afin de ne pas céder aux sirènes de la professionnalisation et à aborder les problématiques de l'activité et des professions adressées à autrui avec le souci d'un profond respect pour les professionnels qui réalisent chaque jour des prodiges humains.

\section{Références bibliographiques :}

Buysse, A. (2011). Une modélisation des régulations et de la médiation dans la construction des savoirs professionnels des enseignants. Dans $P$. Maubant, et S. Martineau, $S$. (dir.) Fondements des pratiques professionnelles des enseignants : pour une lecture compréhensive des fondements des savoirs professionnels des enseignants. Ottawa : Presses Universitaires d'Ottawa.

Jorro, A., \&Ketele, J.-M. (2011). La professionnalité émergente : quelle reconnaissance ? Bruxelles : De Boeck.

Lantheaume, F.\& Helou, C. (2008). La souffrance des enseignants. Une sociologie pragmatique du travail enseignant. Paris : Presses universitaires de France.

Masschelein, J. (2002). Éducation et humanisme. Dans Le Télémaque, 21, p. 37-50. 